\title{
On convergence of solutions of the crystalline Stefan problem with Gibbs-Thomson law and kinetic undercooling
}

\author{
PIOTR RYBKA ${ }^{\dagger}$ \\ Institute of Applied Mathematics, Warsaw University, ul. Banacha 2, 02-097 Warszawa, Poland \\ [Received May 1999 and in revised form 4 January 2000]

\begin{abstract}
This paper presents a study of the relations between the modified Stefan problem in a plane and its quasi-steady approximation. In both cases the interfacial curve is assumed to be a polygon. It is shown that the weak solutions to the Stefan problem converge to weak solutions of the quasi-steady problem as the bulk specific heat tends to zero. The initial interface has to be convex of sufficiently small perimeter.
\end{abstract}

Keywords: Free boundary; Stefan problem; Gibbs-Thomson law; crystalline anisotropy

\section{Introduction}

In this paper we present a study of the connection between the crystalline versions of the modified Stefan problem and its quasi-steady approximation. We assume that the bulk specific heats of a solid and liquid are equal, $e_{\text {solid }}=e_{\text {liquid }}=\epsilon$. We establish convergence of weak solutions to the modified Stefan problem if $\epsilon$ goes to zero. The quasi-steady approximation is obtained by setting $\epsilon$ equal to zero.

We have in mind the crystalline Stefan problem with Gibbs-Thomson relations and kinetic undercooling. That is, we assume that the interface is a polygon. Of course, the Gibbs-Thomson relation has to be suitably reformulated. The system under consideration was derived by Gurtin and Matias [7] as a model for crystal growth. The heat transport in the vessel $\Omega$, the law prescribing the normal velocities $V_{i}$ s of the facets and the Gibbs-Thomson law read as follows (see [7]):

$$
\begin{aligned}
& \epsilon u_{t}^{\epsilon}=\Delta u^{\epsilon} \quad \text { in } \quad \bigcup_{0<t<T}\left(\Omega_{1}(t) \cup \Omega_{2}(t)\right), \\
& \llbracket \nabla u^{\epsilon} \rrbracket v_{j}=-V_{j}^{\epsilon}, \quad j=1, \ldots, N, \\
& \int_{s_{j}^{\epsilon}(t)} u^{\epsilon}=\Gamma_{j}-\beta_{j} L_{j}^{\epsilon}(t) V_{j}^{\epsilon}(t), \quad j=1, \ldots, N,
\end{aligned}
$$

where $N$ is the number of facets $s_{i}$ of the polygonal interface $s(t)$. The notation will be explained in Section 2. We assume that $N$ is constant. We have already shown in [15: Theorem 5.2 (d)] that for small interfaces the isoperimetric quotient decreases. Thus, in this specific situation, this assumption does not seem restrictive. This point of view on splitting facets is supported by M.H. Giga and Y. Giga in [5]. However, their setting is different from ours. Nonetheless, we would like to highlight [5: Lemma 5]. This lemma states that small facets of polygons evolving by a driven

\footnotetext{
Email: rybka@mimuw.edu.pl
} 
crystalline do not split. In general, however, the problem of breaking facets is unanswered; we plan to address this elsewhere.

In $(1.1) \epsilon$ may be positive, leading to the modified Stefan problem, or it may be zero, hence we obtain the quasi-steady approximation.

We remark here that the above problem was formulated by Herring in the metallurgical literature in the 1950s; see [9]. Later, it was independently rediscovered by Ben Amar-Pomeau [2] and Gurtin-Matias [7].

If we augment (1.1) with initial conditions for the distribution of temperature $u$ and position of the interface as well as a boundary condition, (we fix $u=0$ to be the melting temperature), then we may formulate (1.1) in a weak form, (see Section 2). We have already established the local existence and uniqueness for either $\epsilon>0$ or $\epsilon=0$; see [12-15]. The above problem for smooth interfaces is also well-posed. This was established in the early 1990s; see [4] and [11]. It turns out that $\beta>0$ is quite important. The problem for $\beta=0$ and smooth interfaces was studied by Luckhaus [10] and in greater generality by Almgren-Wang [1]. In particular, they showed that uniqueness fails. Uniqueness is an also an open problem if we admit general interfaces for $\beta>0$; see Soner [16].

We shall study here the limit of the weak solution $u^{\epsilon}$ to (1.1). A part of this task is establishing a uniform bound from below for the maximal times of existence $T_{\max }^{\epsilon}$ :

$$
\inf _{\epsilon>0} T_{\max }^{\epsilon} \geqslant T_{0}>0 \text {. }
$$

This is the content of Proposition 3.8 below. Earlier, we established that $u^{\epsilon} \in$ $C^{0, \gamma}\left(\left[0, T_{\max }^{\epsilon}\right), H^{\alpha}(\Omega)\right)$ and $V_{i}^{\epsilon} \in C^{0, \gamma}\left(\left[0, T_{\max }^{\epsilon}\right)\right)$, where $\gamma<\frac{1}{2}, \alpha<\frac{3}{2}$ for $\epsilon>0$ (see [14]) and $\gamma=\frac{1}{2}, \alpha=1$ for $\epsilon=0$ (see [12]).

In the present paper we shall show uniform estimates for $V_{i}^{\epsilon}$ s and $u^{\epsilon}$ in the above-mentioned Hölder norms. They will permit us to prove our main result, Theorem 4.3. It states that

$$
V_{i}^{\epsilon} \rightarrow V_{i}^{0} \quad \text { in } \quad C^{0, \gamma}([0, t]), i=1, \ldots, N \quad u^{\epsilon} \rightarrow u^{0} \quad \text { in } \quad C^{0, \gamma}\left([0, t] ; H^{\alpha}(\Omega)\right),
$$

where $t<T_{0}, \gamma<\frac{1}{2}, \alpha<\frac{3}{2}$ and $\alpha+\gamma<\frac{3}{2}$.

It turns out that feasibility of this program depends very much on $s_{0}$ and $u^{\epsilon}(0)$. We note that $u^{\epsilon}(0)$ may not be arbitrary, but it has to be related to solutions of the quasi-steady approximation; see (3.1) below. We also need $s_{0}$ to be small, convex and not overly deformed from the Wulff shape. We also assume that the Wulff shape is a regular $N$-gon. At present we do not know if it is possible to relax any of this hypotheses, but is seems that our assumption on the Wulff shape is not essential.

This paper is set out as follows. In Section 2 we explain the notation, we recall the weak formulation and its basic properties. In Section 3 we establish the estimate (1.2). In the last section we derive the estimates leading to the convergence result.

\section{Preliminaries}

First of all, we shall complete the description of our problem and the notation. In our system $u$ is the normalized temperature, i.e. it is zero at melting flat interfaces. It is also continuous across the interface. The evolving crystal occupies $\Omega_{1}(t)$, the remaining part, $\Omega_{2}(t)$, of the container $\Omega$ is filled with melt, i.e. $\Omega=\Omega_{1}(t) \cup s(t) \cup \Omega_{2}(t)$, where $s(t)=\partial \Omega_{1}(t) \cap \partial \Omega_{2}(t)$ is the interface. We assume that $\Omega, \Omega_{1}(t), \Omega_{2}(t)$ are bounded regions in $\mathbb{R}^{2}$ and $\Omega_{1}(t) \subset \subset \Omega$. Finally we assume that the boundary $\partial \Omega$ of $\Omega$ is smooth. The $i$ th facet $s_{i}$ of $s$ is determined by its vertices $v_{i}, v_{i+1}$, and $L_{i}=\left|v_{i}-v_{i+1}\right|$ is the length of $s_{i}$. The perimeter $L$ of $s$ is equal to $\sum_{i=1}^{N} L_{i}$. 
We denote by $V_{i}$ the velocity of $s_{i}$ in the direction of the outer normal $v_{i}$. Precisely,

$$
V_{i}(t)=\frac{\mathrm{d}}{\mathrm{d} t} z_{i}(t),
$$

where

$$
z_{i}(t)=\left\{\begin{array}{lll}
\operatorname{dist}\left(l_{i}(t), l_{i}(0)\right) & \text { if } & \left(v_{i}(t)-v_{i}(0)\right) \cdot v_{i}>0, \\
-\operatorname{dist}\left(l_{i}(t), l_{i}(0)\right) & \text { if } & \left(v_{i}(t)-v_{i}(0)\right) \cdot v_{i}<0,
\end{array}\right.
$$

and $l_{i}(t)$ is the line containing $s_{i}(t)$. The definition of $V_{i}$ in (1.1) involves the jump $\mathbb{I} \cdot \|$ across $s(t)$. This quantity is given by

$$
\llbracket \phi \rrbracket\left(x_{0}\right)=\lim _{\Omega_{2}(t) \ni x \rightarrow x_{0}} \phi(x)-\lim _{\Omega_{1}(t) \ni x \rightarrow x_{0}} \phi(x), \quad x_{0} \in s(t)=\partial \Omega_{1}(t) \cap \partial \Omega_{2}(t) .
$$

We shall consider only admissible polygonal interfaces. Admissibility means here that the outer normals $v_{i}$ to the facets $s_{i}$ belong to the set $\mathcal{S}$ of normals of a given Wulff shape $W$ (cf. Sections 7 and 12 in [6]). Moreover, we require that normals to successive facets in $s$, must be neighbouring normals to $W$. For the sake of the present analysis we may think of $W$ as being a given convex polygon with $M$ edges numbered counterclockwise. Let us note that $N \geqslant M$ and the equality holds if $s$ is convex.

The kinetic coefficients $\beta_{j}>0$ are constants, so are $\Gamma_{j}, j=1, \ldots, N$, and they are defined depending on $s$ as follows (see Section 12.5 in [6]):

$$
\Gamma_{j}= \begin{cases}-\ell_{j} & \text { if } s \text { is locally convex near both vertices } v_{j}, v_{j+1}, \\ \ell_{j} & \text { if } s \text { is locally concave near both vertices } v_{j}, v_{j+1}, \\ 0 & \text { otherwise, }\end{cases}
$$

where $\ell_{j}$ is the length of the edge of the Wulff shape with normal $v_{j}$.

Let us note that $\Gamma_{j}$ is closely related to the underlying interface energy density $f$ (which is basically defined on the unit circle). This is due to the fact that $f$ enters the definition of the Wulff shape $W$ (see Section 7 of [6] and especially Subsection 7.5). It follows from this definition that if $d_{i}$ is the distance from the origin to the $i$ th edge of $W$, then

$$
d_{i}=f\left(v_{i}\right),
$$

where $v_{i}$ is the outer normal to the $i$ th edge of $W$.

Interestingly, $\Gamma_{j} / L_{j}$ is the crystalline weighted curvature of $s_{j}$. The relevant definition, which does not need any differential structure of $s$, is given in [17: p. 423]. We will recall it here. Let us suppose that $z_{i}$ s are as defined in (2.1) and $\mathbf{z}=\left(z_{1}, \ldots, z_{N}\right)$, i.e. $s(\mathbf{z})$ is a polygon resulting from $s$ by moving entire facet $s_{i}$ by $z_{i}$ in the direction of the normal $v_{i}, A(\mathbf{z})$ is the area surrounded by $s(\mathbf{z})$ and $L_{j}(\mathbf{z})$ is the length of $j$ th facet of $s(\mathbf{z})$. If we set the surface energy $E(\mathbf{z})=\sum_{i=1}^{N} f\left(v_{i}\right) L_{i}(\mathbf{z})$, then we define the crystalline weighted curvature $\mathcal{K}_{i}$ of $s_{i}$ as

$$
\mathcal{K}_{i}=-\lim _{\Delta z_{i} \rightarrow 0} \frac{E\left(\mathbf{z}+\mathbf{e}_{i} \Delta z_{i}\right)-E(\mathbf{z})}{A\left(\mathbf{z}+\mathbf{e}_{i} \Delta z_{i}\right)-A(\mathbf{z})}
$$

where $\mathbf{e}_{i}, i=1, \ldots, N$ are the standard unit vectors of the coordinate axis in $\mathbb{R}^{N}$. This limit may be evaluated with the aid of the lemma below the proof of which we leave to the reader (cf. also [13]). 
LEMMA 2.1 Let us suppose we are given a polygon $s$ with its edges $s_{i}$ numbered counterclockwise, $i=1, \ldots, N$. If $L_{i}$ is the length of edge $s_{i}$ and $\theta_{i}$ is the (oriented) angle between normals $v_{i-1}$ and $v_{i}$ to $s_{i-1}$ and $s_{i}$, respectively, then

$$
\begin{aligned}
\Delta L_{i}: & =L_{i}(\mathbf{z}+\Delta \mathbf{z})-L_{i}(\mathbf{z}) \\
& =-\Delta z_{i}\left(\operatorname{ctan} \theta_{i}+\operatorname{ctan} \theta_{i+1}\right)+\frac{\Delta z_{i-1}}{\sin \theta_{i}}+\frac{\Delta z_{i+1}}{\sin \theta_{i+1}},
\end{aligned}
$$

where $\Delta \mathbf{z}=\left(\Delta z_{1}, \ldots, \Delta z_{N}\right)$. If we further assume that $s$ is a convex polygon, the origin belongs to the region bounded by $s$ and $d_{i}$ is the distance from the origin to $s_{i}$, then

$$
L_{i}=-d_{i}\left(\operatorname{ctan} \theta_{i}+\operatorname{ctan} \theta_{i+1}\right)+\frac{d_{i-1}}{\sin \theta_{i}}+\frac{d_{i+1}}{\sin \theta_{i+1}} .
$$

Here, by convention, $s_{N+1}=s_{1}$, etc.

It is not difficult to check now that $\mathcal{K}_{i}=\Gamma_{i} / L_{i}$ (see also [17]). We note that the formula for $\mathcal{K}_{i}$ is particularly simple for convex admissible polygons and Wulff shapes, being a regular $N$-gons. Namely, we have

$$
\mathcal{K}_{i}=\frac{d \kappa}{L_{i}}<0, \quad i=1, \ldots, N=M
$$

where $d$ is the distance of any facet to the centre of symmetry of $W$ and

$$
\kappa=2 \operatorname{ctan} \theta-\frac{2}{\sin \theta}=-2 \tan \frac{\pi}{N} .
$$

It is clear that the above definition applies only to polygons. Readers who are interested in evolution by curvature of more general sets is referred to a recent paper by Bellettini et al., [3: Section 3].

In order to obtain a closed system we augment equations (1.1) with initial and boundary data. We consider here only homogeneous Dirichlet boundary data

$$
\left.u\right|_{\partial \Omega}=0 \quad \text { for } t \geqslant 0 .
$$

This choice gives us some technical advantages. We shall not consider the Neumann condition, which is physically relevant, because our tools do not apply directly to it.

We impose the initial conditions

$$
u(0, x)=u_{0}(x), \quad s(0)=s_{0} .
$$

We remark that if $\epsilon=0$, then we do not specify the initial distribution of temperature, because it is a part of a solution.

In $[12,14]$ we defined a weak solution of $(1.1)$ on $[0, T)$ as a pair $\left(\mathbf{z}^{\epsilon}, u^{\epsilon}\right)$ where $\mathbf{z}^{\epsilon}$ is as in (2.1) and $\mathbf{z}^{\epsilon} \in C^{1}\left([0, T) ; \mathbb{R}^{N}\right), \mathbf{z}^{\epsilon}(0)=0, u^{\epsilon} \in C^{\alpha}\left([0, T), H_{0}^{1}(\Omega)\right)$ with $u(0)=u_{0}$, $u_{t} \in L_{\text {loc }}^{\infty}\left([0, T), H^{-1}(\Omega)\right.$ ) (where $H^{-1}(\Omega)$ is the dual of $H_{0}^{1}(\Omega)$ ). This condition on $u_{t}$ is imposed only if $\epsilon>0$. Finally, the identities

$$
\begin{gathered}
\epsilon\left\langle u_{t}, h\right\rangle=-\int_{\Omega} \nabla u(t, x) \cdot \nabla h(x) \mathrm{d} x+\sum_{j=1}^{N} \int_{s_{j}(t)} V_{j}^{\epsilon}(t) h(x) \mathrm{d} l, \quad \forall h \in H_{0}^{1}(\Omega), \\
\int_{s_{j}^{\epsilon}(t)} u \mathrm{~d} l=\Gamma_{j}-\beta_{j} L_{j}^{\epsilon}(t) V_{j}^{\epsilon}(t), \quad j=1, \ldots, N
\end{gathered}
$$


hold, where $\langle\cdot, \cdot\rangle$ is the pairing between $H^{-1}(\Omega)$ and $H_{0}^{1}(\Omega)$. We shall call (2.3a) and (2.3b) problem $\left(\mathrm{P}_{\epsilon}\right)$ for $\epsilon>0$ and problem $\left(\mathrm{P}_{0}\right)$ if $\epsilon=0$.

The existence and uniqueness of a weak solution $\left(\mathbf{z}^{\epsilon}, u^{\epsilon}\right)$ of $\left(\mathrm{P}_{\epsilon}\right)$ (respectively, weak solution $\left(\mathbf{z}^{0}, u^{0}\right)$ of $\left.\left(\mathrm{P}_{0}\right)\right)$ on a maximal interval of existence $\left[0, T_{\max }^{\epsilon}\right)$ (respectively, on $\left.\left[0, T_{\max }^{0}\right)\right)$ has been shown in $[14,15]$, (respectively, $[12,13])$. We stress that a global existence result cannot be expected, especially if we fix the number of edges. This is because topological catastrophes, for example self- intersection, collapsing of a facet to a point and bumping into the boundary, would be imminent.

Notation. Throughout the paper vector quantities are set in bold, e.g. $\mathbf{z}=\left(z_{1}, \ldots, z_{N}\right)$, the inner product in $\mathbb{R}^{k}$ is denoted by dot: $\mathbf{a} \cdot \mathbf{b}=\sum_{i=1}^{k} a_{i} b_{i},|\mathbf{a}|$ is the Euclidean norm $|\mathbf{a}|^{2}=\mathbf{a} \cdot \mathbf{a}$. For $a \in \mathbb{R}^{N}$ we set $|a|_{1}=\sum_{i=1}^{N}\left|a_{i}\right|$. For the sake of brevity we shall write $\mathbf{G}$ for $\left(\Gamma_{1} / \beta_{1}, \ldots, \Gamma_{N} / \beta_{N}\right)$ and $\mathbf{B}$ for $\left(\beta_{1}, \ldots, \beta_{N}\right)$. Finally, $(f, g)_{H_{0}^{1}(\Omega)}$ is the inner product in $H_{0}^{1}(\Omega)$, i.e. $(f, g)_{H_{0}^{1}(\Omega)}=$ $\int_{\Omega} \nabla f(x) \cdot \nabla g(x) \mathrm{d} x$ and $\|f\|^{2}=(f, f)_{H_{0}^{1}(\Omega)}$.

\section{Uniform estimates for the extinction time}

We provide in $\epsilon$ uniform lower bounds for the maximal time of existence. The method we use depends essentially on the fact that $s_{0}$ is convex, small and the isoperimetric quotient of $s_{0}$ is not large either. At present it is not clear to us how to relax these assumptions.

We first recall some facts about the structure of the solution and the evolution. We also give a new proof of $u^{\epsilon}(t)<0$ for $\epsilon>0$. This was shown in [15], but here we use a different method which clearly has the virtue of being independent of $\epsilon>0$. It turns out that the negativity of $u^{\epsilon}(t)$, combined with the smallness of $L^{\epsilon}(t)$, leads to estimates of the velocity of facets, namely $\left|V_{i}^{\epsilon}\right| \leqslant\left|\Gamma_{i}\right| / \beta_{i} L_{i}^{\epsilon}$. This inequality indicates that the velocity blows up but in a controllable manner. This inequality suffices to show that $T_{\max }^{\epsilon} \geqslant T_{0}>0$.

Our analysis depends very much on the geometric estimates as in [15: Theorem 4.1], which will be recalled below. This theorem was shown under the assumption

$$
\text { the Wulff shape is a regular } N \text {-sided polygon. }
$$

This result was subsequently applied to the flow of $\left(\mathrm{P}_{\epsilon}\right)$, but under an additional simplifying assumption, namely,

$$
\beta_{i} \equiv \beta>0, \quad i=1, \ldots, N .
$$

However, hypotheses $(\mathrm{W})$ and $(\beta)$ play no role in our analysis below. Strictly speaking, our results are valid only if $(\mathrm{W})$ and $(\beta)$ hold, but we state them in a such way that no changes will be required if a generalization of [15: Theorem 4.1] becomes available.

It is important for our convergence result that the initial data for $\left(\mathrm{P}_{\epsilon}\right)$ are consistent with $\left(\mathrm{P}_{0}\right)$. Otherwise, it will not be possible to obtain continuous functions in the limit. We have shown (see [13: equation (13)] as well as the definition of weak solutions above) that if $\left(\mathbf{z}^{0}, u^{0}\right)$ is a weak solution to $\left(\mathrm{P}_{0}\right)$, then

$$
u^{0}(t)=\sum_{i=1}^{N} V_{i}^{0}(t) f_{i}\left(\mathbf{z}^{0}(t)\right)
$$


where $f_{i}(\mathbf{z})$ will be defined below. It is clear from this formula that the initial value of temperature may not be specified. Thus, we shall call the data for $\left(\mathrm{P}_{\epsilon}\right)$ consistent with $\left(\mathrm{P}_{0}\right)$ provided that the initial temperature distribution $u^{\epsilon}(0)$ satisfies

$$
u^{\epsilon}(0) \equiv u_{0} \equiv u^{0}(0)=\sum_{i=1}^{N} V_{i}^{0}(0) f_{i}(0) .
$$

Now, we shall recall the definition of $f_{i}$. Let us suppose that $s_{i}=s_{i}(\mathbf{z})$ is a facet of $s=$ $s(\mathbf{z})$. Then, due to the Riesz Representation Theorem, $f_{i} \equiv f_{i}(\mathbf{z})$ is the unique element of $H_{0}^{1}(\Omega)$ satisfying (see $[12,14])$

$$
\int_{s_{i}} h \mathrm{~d} l=\left(h, f_{i}\right)_{H_{0}^{1}(\Omega)}, \quad \forall h \in H_{0}^{1}(\Omega) .
$$

As a matter of fact $f_{i}$ is smoother than just $H_{0}^{1}(\Omega)$. The proper scale of smoothness is provided by fractional Sobolev spaces or fractional power of $L^{2}(\Omega)$. We will now comment on these spaces. Our first observation is expressed below.

LemMA 3.1 ( [8: Section 1.6], [15: equation (3.5)]) Let us set $X=L^{2}(\Omega)$. We define $\mathcal{A}: D(\mathcal{A}) \subset$ $X \rightarrow X$ by formula $\mathcal{A} u=-\Delta u$, and $D(\mathcal{A})=H^{2}(\Omega) \cap H_{0}^{1}(\Omega)$. Then, $\mathcal{A}$ is a self-adjoint operator, moreover $\mathcal{A}$ is positive definite, i.e.

$$
\sigma(\mathcal{A}) \geqslant \lambda>0
$$

and hence $\mathcal{A}$ is sectorial. Thus, the spaces $X^{\alpha}, \alpha \geqslant 0$ are well defined, in particular we have

$$
X^{1 / 2}=H_{0}^{1}(\Omega) \text {. }
$$

The fact that $\mathcal{A}$ is sectorial implies that $\mathrm{e}^{t \Delta}$ is an analytic semi-group; (see [8]). In the present work we will use various bounds on $(-\Delta)^{\alpha} \mathrm{e}^{t \Delta}$ (see [8: Chapter I]), as well as the integral representation of the solution. Namely, we have

$$
u^{\epsilon}(t)=\mathrm{e}^{\Delta t / \epsilon} u_{0}-\frac{1}{\epsilon} \sum_{i=1}^{N} \int_{0}^{t} \Delta \mathrm{e}^{\Delta(t-\tau) / \epsilon} f_{i}\left(\mathbf{z}^{\epsilon}(\tau)\right) V_{i}^{\epsilon}(\tau) \mathrm{d} \tau .
$$

This is formula (5.6) in [15] which is at the very bottom of $\mathrm{p} \mathrm{780;} \mathrm{it} \mathrm{also} \mathrm{appears} \mathrm{in} \mathrm{[14]} \mathrm{right} \mathrm{after}$ equation (4.5). However, in [14] the factor $\frac{1}{\epsilon}$ in front of the integral was erroneously omitted.

Let us now complete the discussion of smoothness of $f_{i}$. We recall that if $s$ is a polygon which is separated away from the boundary of $\Omega$, then (see [13: Lemma 9] and [14: Lemma 3.3])

$$
\left\|f_{i}\right\|_{H_{0}^{1}(\Omega)} \leqslant C_{0} L_{i}\left|\ln L_{i}\right|^{1 / 2}, \quad\left\|f_{i}\right\|_{X^{\alpha / 2}} \leqslant C_{0} L_{i}^{\gamma},
$$

where $\alpha \in\left(1, \frac{3}{2}\right)$ and $\gamma \in\left(0, \frac{1}{2}\right)$. The constant $C_{0}$ depends upon the diameter of $\Omega$ and the distance of $s$ to $\partial \Omega$.

Moreover, if $s_{1}, s_{2}$ are two admissible polygons such that $s_{2} \equiv s_{2}(\Delta \mathbf{z})$ is formed from $s_{1}$ by moving the $i$ th facet of $s_{1}$ in the normal direction by $\Delta z_{i}$, then (see [14: Lemma 3.4])

$$
\left\|f_{i}\left(s_{1}\right)-f_{i}\left(s_{2}\right)\right\|_{X^{\alpha / 2}} \leqslant C_{1}|\Delta \mathbf{z}|^{\gamma},
$$


where $\alpha \in\left(1, \frac{3}{2}\right), \gamma \in\left(0, \frac{1}{2}\right)$ and $\alpha+\gamma<\frac{3}{2}$. The constant $C_{1}$ depends upon the diameter of $\Omega$ and the distance of $s_{1}, s_{2}$ to $\partial \Omega$. Since our subsequent analysis deals with polygons $s$ whose distance to $\partial \Omega$ is bounded below by a positive number, then we may treat $C_{i}, i=1,2$ in (3.5), (3.6) as independent of $s, s_{1}, s_{2}$.

Now, we will recall some simple facts about the solutions to $\left(\mathrm{P}_{\epsilon}\right)$ and $\left(\mathrm{P}_{0}\right)$. We shall start with remarks on small consistent data.

Proposition 3.2 Let us suppose that $s_{0}$ is convex and satisfies hypothesis (a) of [13: Theorem 10]. We set $\Lambda=K:=\max _{i=1, \ldots, N} L_{0 i} / \min _{i=1, \ldots, N} L_{0 i}$. If we further assume that $L(0)$ is small, i.e. hypothesis (e) of [13: Theorem 10] is fulfilled, then

$$
V_{i}^{0}(0)<0 .
$$

Proof. This is in fact established in [13: Theorem 10, (i)].

If we recall that $f_{i}(\mathbf{z})>0$ (see [13: Lemma 2]) then we immediately obtain the following.

COROLlaRY 3.3 Let us suppose that $s_{0}$ is as in the previous proposition and $\left(\mathbf{z}^{0}, u^{0}\right)$ is a unique solution of $\left(\mathrm{P}_{0}\right)$. Then

$$
u^{0}(0)=\sum_{i=1}^{N} f_{i}(0) V_{i}^{0}(0)<0 .
$$

We now gather simple but important estimates for solutions of $\left(\mathrm{P}_{\epsilon}\right)$.

Proposition 3.4 Let us suppose that $\left(\mathbf{z}^{\epsilon}, u^{\epsilon}\right)$ is a unique solution to $\left(\mathrm{P}_{\epsilon}\right)$ with initial data $u_{0}$. If $V_{i}^{\epsilon}(t)<0, i=1, \ldots, N, t \in[0, T]$ and $u_{0} \leqslant 0$, then $u^{\epsilon}(t)<0$ for $t \in[0, T]$.

Proof. This follows immediately from [14: Lemma 4.5] which states that $-\Delta \mathrm{e}^{\Delta t} f_{i} \geqslant 0$ for $t>0$ and from the representation formula (3.4).

Proposition 3.5 (cf. [13: Proposition 8]) If $s^{\epsilon}(t)$ is convex, $V_{i}^{\epsilon}(t)<0, i=1, \ldots, N$ and $u^{\epsilon}(t)<0$, then

$$
\left|V_{i}^{\epsilon}\right| \leqslant \frac{\left|\Gamma_{i}\right|}{\beta_{i} L_{i}^{\epsilon}} .
$$

Proof. By assumption

$$
\int_{s_{i}^{\epsilon}} u^{\epsilon} \mathrm{d} l<0
$$

hence

$$
0<-L_{i}^{\epsilon} \beta_{i} V_{i}^{\epsilon}=-\Gamma_{i}+\int_{s_{i}^{\epsilon}} u^{\epsilon} \mathrm{d} l<-\Gamma_{i} .
$$

We close these remarks by noting the following.

COROLlARY 3.6 Let us suppose that the assumptions of the previous proposition hold. Then,

$$
\frac{\mathrm{d} L^{\epsilon}}{\mathrm{d} t}(t)<0 .
$$


Proof. Lemma 2.1 implies that

$$
\frac{\mathrm{d} L^{\epsilon}}{\mathrm{d} t}(t)=-\sum_{i=1}^{N} \kappa_{i} V_{i}^{\epsilon}(t) .
$$

Due to convexity of $s^{\epsilon}(t)$ we have $\kappa_{i}<0, i=1, \ldots, N$. Hence, $\frac{\mathrm{d} L^{\epsilon}}{\mathrm{d} t}(t)<0$.

Proposition 3.5 yields estimates for $\left|V_{i}^{\epsilon}\right|$. Thus, we have to make sure that its hypothesis holds. In fact we showed this in [13-15] but the structure of the proof for $\epsilon>0$ suggests that it may depend on $\epsilon$. Below we present a new proof which is free from this deficiency. But first we have to point out the geometric background, which is [15: Theorem 4.1]. If we denote by $Q(s)$ the isoperimetric quotient of a polygon $s$, i.e. $Q(s)=L^{2} / A$, then this theorem guarantees existence of two positive constants $\eta$ and $\Lambda$ such that if

$$
Q(s)<Q(W)+\eta,
$$

then

$$
\frac{\max _{i=1, \ldots, N} L_{i}}{\min _{i=1, \ldots, N} L_{i}}<\Lambda,
$$

where $W$ is the Wulff shape. This theorem was shown under the assumption (W), but the question of its generalizations is unanswered.

Before stating our theorem let us introduce some more notation. For $\delta, \lambda>0$ we shall write

$$
K_{0}(\lambda, \delta):=\lambda^{-\delta} \int_{0}^{\infty} \tau^{-1+\delta} \mathrm{e}^{-\tau} \mathrm{d} \tau=\lambda^{-\delta} \Gamma(\delta)
$$

where $\Gamma(t)$ is Euler's gamma function.

THEOREM 3.7 Let us suppose that $s_{0}$ is convex, (3.7) is fulfilled and $u^{\epsilon}(0)$ is consistent with $\left(\mathrm{P}_{0}\right)$. We fix arbitrary numbers $\delta \in\left(0, \frac{1}{4}\right), \gamma \in\left(0, \frac{1}{2}\right)$. Then, there exists a positive constant $K_{1}$, which is independent of $s_{0}$, and it has the following property. If we assume that $L^{\epsilon}(0)$ is small, meaning that hypothesis (e) of [13: Theorem 10] is fulfilled with $K=\Lambda$, and

$$
K_{1}(N \Lambda)^{1-\gamma}|\mathbf{G}|_{1}\left(1+K_{0}(\lambda, \delta)\right)\left(L^{\epsilon}(0)\right)^{\gamma}\left|\ln \left(L^{\epsilon}(0) / N \Lambda\right)\right|^{1 / 2} \leqslant \min _{i=1, \ldots, N}\left|\Gamma_{i}\right|,
$$

holds, then $u^{\epsilon}(t)<0$ and $V_{i}^{\epsilon}(t)<0, i=1, \ldots, N$ for $t \in\left[0, T_{\max }^{\epsilon}\right)$.

Proof. We introduce a set $E \subset\left[0, T_{\max }^{\epsilon}\right)$,

$$
E=\left\{t \in\left[0, T_{\max }^{\epsilon}\right): \forall \tau \in[0, t], \quad V_{i}^{\epsilon}(\tau)<0, \quad i=1, \ldots, N \quad \text { and } \quad u^{\epsilon}(\tau)<0\right\} .
$$

Obviously, by Proposition 3.2 and Corollary 3.3, 0 is in $E$. Moreover, by continuity of $V_{i}^{\epsilon}(\tau)$ we infer that $E \neq\{0\}$. Hence $\mu:=\sup E>0$. Our goal is to show that $\mu=T_{\max }^{\epsilon}$. Let us assume otherwise. Our calculations are based on a representation of $u^{\epsilon}$ provided by formula (3.4). Let us now fix an arbitrary number $\alpha \geqslant 1$ such that $\alpha+2 \delta<\frac{3}{2}$. It follows that

$$
\begin{aligned}
& \left\|u^{\epsilon}(t)\right\|_{X^{\alpha / 2}} \leqslant C\left\|u_{0}\right\|_{X^{\alpha / 2}} \\
& \quad+\int_{0}^{t} \frac{1}{\epsilon}\left\|(-\Delta)^{1-\delta} \mathrm{e}^{\Delta(t-\theta) / \epsilon} \sum_{i=1}^{N}(-\Delta)^{\alpha / 2+\delta} f_{i}\left(\mathbf{z}^{\epsilon}(\theta)\right) V_{i}^{\epsilon}(\theta)\right\|_{L^{2}(\Omega)} \mathrm{d} \theta .
\end{aligned}
$$


With the help of [8: Theorem 1.4.3] and Lemma 3.1 for $t<\mu$ we arrive at

$$
\begin{aligned}
& \left\|u^{\epsilon}(t)\right\|_{X^{\alpha / 2}} \leqslant C\left\|u_{0}\right\|_{X^{\alpha / 2}} \\
& \quad+\int_{0}^{t} \frac{C}{\epsilon}\left(\frac{t-\theta}{\epsilon}\right)^{-1+\delta} \mathrm{e}^{-\lambda(t-\theta) / \epsilon} \sum_{i=1}^{N}\left\|f_{i}\left(\mathbf{z}^{\epsilon}(\theta)\right)\right\|_{X^{\alpha / 2+\delta}}\left|V_{i}^{\epsilon}(\theta)\right| \mathrm{d} \theta .
\end{aligned}
$$

Now, due to (3.5), Proposition 3.5 and after a change of variable we obtain that

$$
\begin{aligned}
\left\|u^{\epsilon}(t)\right\|_{X^{\alpha / 2}} \leqslant & C\left\|u_{0}\right\|_{X^{\alpha / 2}} \\
& +C \lambda^{-\delta} \int_{0}^{t / \epsilon} \tau^{-1+\delta} \mathrm{e}^{-\tau} \sum_{i=1}^{N}\left(L_{i}^{\epsilon}\right)^{\gamma-1}(t-\epsilon \tau / \lambda) \frac{\left|\Gamma_{i}\right|}{\beta_{i}} \mathrm{~d} \tau .
\end{aligned}
$$

We now recall that our assumptions guarantee that (3.8) holds. If we keep this in mind we notice a series of simple inequalities which we will use frequently:

$$
L(t) / 2 \geqslant L_{i}(t) \geqslant \min _{i=1, \ldots, N} L_{i}(t) \geqslant \max _{i=1, \ldots, N} L_{i}(t) / \Lambda \geqslant L(t) /(N \Lambda) .
$$

Hence (3.5), (3.10), Corollary 3.6 and the consistency of $u_{0}$ with $\left(\mathrm{P}_{0}\right)$ imply that

$$
\left\|u^{\epsilon}(t)\right\|_{X^{\alpha / 2}} \leqslant C_{2}(N \Lambda)^{1-\gamma}|\mathbf{G}|_{1}\left[\left(L^{\epsilon}(0)\right)^{\gamma-1}+K_{0}(\lambda, \delta)\left(L^{\epsilon}(t)\right)^{\gamma-1}\right] .
$$

We are now in a position to estimate $\left|\int_{s_{i}^{\epsilon}} u^{\epsilon} \mathrm{d} l\right|$. We want to show that this is small,

$$
\begin{aligned}
\left|\int_{s_{i}^{\epsilon}} u^{\epsilon} \mathrm{d} l\right| & =\left|\left(u^{\epsilon}, f_{i}\left(\mathbf{z}^{\epsilon}\right)\right)_{H_{0}^{1}(\Omega)}\right| \leqslant\left\|u^{\epsilon}\right\|_{H_{0}^{1}(\Omega)}\left\|f_{i}\left(\mathbf{z}^{\epsilon}\right)\right\|_{H_{0}^{1}(\Omega)} \\
& \leqslant C_{0}\left\|u^{\epsilon}\right\|_{X^{\alpha / 2}} L_{i}^{\epsilon}(t)\left|\ln L_{i}^{\epsilon}(t)\right|^{1 / 2}
\end{aligned}
$$

where we applied (3.5) and $\alpha \geqslant 1$.

Combining this inequality with (3.11) yields

$$
\begin{aligned}
\left|\int_{s_{i}^{\epsilon}} u^{\epsilon}(t) \mathrm{d} l\right| \leqslant & C_{0} C_{2}(N \Lambda)^{1-\gamma}|\mathbf{G}|_{1}\left(L^{\epsilon}(0)\right)^{-1+\gamma} L^{\epsilon}(t)\left|\ln \left(L^{\epsilon}(t) / N \Lambda\right)\right|^{1 / 2} \\
& +C_{0} C_{2}(N \Lambda)^{1-\gamma}|\mathbf{G}|_{1} K_{0}(\lambda, \delta)\left(L^{\epsilon}(t)\right)^{\gamma}\left|\ln \left(L^{\epsilon}(t) / N \Lambda\right)\right|^{1 / 2},
\end{aligned}
$$

where the constant $C_{2}$ is independent of $s(t)$.

Due to the continuity of both sides of the above expression, and $t<\mu<T_{\max }^{\epsilon}$, this inequality holds also for $t=\mu$. But now Corollary 3.6 and the monotonicity of the function $x \mapsto x^{\xi}|\ln x|^{1 / 2}$ for $\xi>0$ on $[0, \sqrt{e}]$ imply that for $K_{1}=C_{0} C_{2}$

$$
\left|\int_{s_{i}^{\epsilon}(\mu)} u^{\epsilon}(\mu) \mathrm{d} l\right|<K_{1}(N \Lambda)^{1-\gamma}|\mathbf{G}|_{1}\left(1+K_{0}(\lambda, \delta)\right)\left(L^{\epsilon}(0)\right)^{\gamma}\left|\ln \left(L^{\epsilon}(0) / N \Lambda\right)\right|^{1 / 2} .
$$

Thus, due to the smallness of $L^{\epsilon}(0)$ we infer that for all $i=1, \ldots, N$

$$
V_{i}^{\epsilon}(\mu)=\left(\Gamma_{i}-\int_{s_{i}^{\epsilon}(\mu)} u(\mu) \mathrm{d} l\right) /\left(\beta_{i} L_{i}^{\epsilon}(\mu)\right)<\left(\Gamma_{i}+\left|\Gamma_{i}\right|\right) /\left(\beta_{i} L_{i}^{\epsilon}(\mu)\right)=0 .
$$

The inequality above, the continuity of $V_{i}^{\epsilon}$ and Proposition 3.4 mean that we have reached a contradiction. Thus, $\mu=T_{\max }^{\epsilon}$. 
This theorem and Proposition 3.5 immediately imply the following inequality:

$$
\left|V_{i}^{\epsilon}\right| \leqslant \frac{\left|\Gamma_{i}\right|}{\beta_{i} L_{i}^{\epsilon}} .
$$

Of course the right-hand side of estimate (3.12) blows up, nonetheless it is sufficient to show a uniform bound from below on $T_{\max }^{\epsilon}$. This will be accomplished in the next proposition, but first we describe the setting. We fix an admissible polygon $s_{0}$ and we consider a ball $B(0, r)$ in $\mathbb{R}^{N}$ whose points correspond to polygons nearby to $s_{0}$. We assume that the radius $r$ has the following properties:

(a) if $\mathbf{z} \in B(0, r)$, then $L_{i}(\mathbf{z}) / L_{i}(0) \in\left[\frac{1}{2}, 2\right]$;

(b) if $\mathbf{z}_{1}, \mathbf{z}_{2} \in B(0, r)$, then $\left\|f_{i}\left(\mathbf{z}_{1}\right)-f_{i}\left(\mathbf{z}_{2}\right)\right\|_{X^{\alpha / 2}} \leqslant C\left|\mathbf{z}_{1}-\mathbf{z}_{2}\right|^{\gamma}, i=1, \ldots, N$, where $C$ is independent of $\mathbf{z}_{1}, \mathbf{z}_{2}$ and $\alpha \in\left[1, \frac{3}{2}\right), \gamma \in\left(0, \frac{1}{2}\right)$, obeying $\alpha+\gamma<\frac{3}{2}$, are fixed;

(c) if $\mathbf{z}_{1}, \mathbf{z}_{2} \in B(0, r)$, then $\left|L_{i}^{-1}\left(\mathbf{z}_{1}\right)-L_{i}^{-1}\left(\mathbf{z}_{2}\right)\right| \leqslant C\left|\mathbf{z}_{1}-\mathbf{z}_{2}\right|, i=1, \ldots, N$ for $C$ independent of $\mathbf{z}_{1}, \mathbf{z}_{2}$.

Our result is then produced as follows.

PROPOSITION 3.8 Let us suppose that the assumptions of the previous theorem hold. We choose $r>0$ such that $(\mathrm{a}-\mathrm{c})$ above are fulfilled. We further assume that for $u_{0}(3.1)$ holds. Then, there is $T_{0}>0$ such that

$$
\inf _{\epsilon>0} T_{\max }^{\epsilon} \geqslant T_{0}>0 .
$$

Proof. Let us define $T_{d}^{\epsilon}$ as the largest $T$ such that for all $t \in[0, T]$ we have $\mathbf{z}^{\epsilon}(t) \in B(0, r)$. Our goal is to relate $r$ to $T_{d}^{\epsilon}$. We have

$$
z_{i}^{\epsilon}(t)=\int_{0}^{t} \frac{\mathrm{d} z_{i}^{\epsilon}}{\mathrm{d} \tau}(\tau) \mathrm{d} \tau=\int_{0}^{t} V_{i}^{\epsilon}(\tau) \mathrm{d} \tau .
$$

Hence (3.12) and (c) imply that for $t \leqslant T_{d}^{\epsilon}$ we have

$$
\begin{aligned}
\left|z_{i}^{\epsilon}(t)\right| & \leqslant \frac{\left|\Gamma_{i}\right|}{\beta_{i}} \int_{0}^{t}\left(\left|1 / L_{i}^{\epsilon}(\tau)-1 / L_{i}^{\epsilon}(0)\right|+1 / L_{i}^{\epsilon}(0)\right) \mathrm{d} \tau \\
& \leqslant \frac{C\left|\Gamma_{i}\right|}{\beta_{i}} \int_{0}^{t}\left|\mathbf{z}^{\epsilon}(\tau)\right| \mathrm{d} \tau+\frac{\left|\Gamma_{i}\right| t}{\beta_{i} L_{i}^{\epsilon}(0)} \leqslant \frac{\left|\Gamma_{i}\right| C t}{\beta_{i}} \max _{\tau \in[0, t]}\left|\mathbf{z}^{\epsilon}(\tau)\right|+\frac{\left|\Gamma_{i}\right| t}{\beta_{i} L_{i}^{\epsilon}(0)} .
\end{aligned}
$$

If we set

$$
\left\|\mathbf{z}^{\epsilon}\right\|:=\sqrt{\max _{\tau \in\left[0, T_{d}^{\epsilon}\right]} \sum_{i=1}^{N}\left|z_{i}^{\epsilon}(\tau)\right|^{2}},
$$

then the above inequality may be rewritten as

$$
\left\|\mathbf{z}^{\epsilon}\right\| \leqslant t|\mathbf{G}|\left(C\left\|\mathbf{z}^{\epsilon}\right\|+\sqrt{\sum_{i=1}^{N}\left(L_{i}^{\epsilon}(0)\right)^{-2}}\right) .
$$

If we now set

$$
T_{0}:=\frac{r}{|\mathbf{G}|\left(C r+\sqrt{\sum_{i=1}^{N}\left(L_{i}^{\epsilon}(0)\right)^{-2}}\right)},
$$


then due to the definition of $T_{d}^{\epsilon}$, we have $T_{0} \leqslant T_{d}^{\epsilon}$ and

$$
\mathbf{z}^{\epsilon}(t) \in B(0, r), \quad \text { for all } \quad t \in\left[0, T_{0}\right] .
$$

Our claim follows.

By the very definition of $r$ and $T_{0}$ we have

COROLlary 3.9

$$
\frac{L^{\epsilon}\left(T_{0}\right)}{L^{\epsilon}(0)} \geqslant \frac{1}{2}
$$

\section{Convergence}

We have shown that solutions to $\left(\mathrm{P}_{\epsilon}\right)$ and $\left(\mathrm{P}_{0}\right)$ are Hölder continuous in time (see [12] and [14], respectively). Our present goal is to show that these estimates are uniform in $\epsilon$. This is true only for small convex initial interfaces $s_{0}$ whose isoperimetric quotient $Q\left(s_{0}\right)$ satisfies (3.7). We have to impose this restriction in order to guarantee that (3.8) holds, and we rely on this estimate.

Moreover, the initial condition for $u^{\epsilon}$ has to be consistent with $\left(\mathrm{P}_{0}\right)$ (see (3.1)). Once we establish the uniform estimates we then subsequently use a compactness argument.

The consistency will manifest itself below in a simplification of (3.4). Furthermore it plays an essential role in the proof of convergence. It will be seen that the uniform estimates on $V_{i}^{\epsilon}$ s in Hölder norm fail for different initial conditions on $u^{\epsilon}$. Thus, without the consistency condition (3.1) it is not possible to show that the limit of $u^{\epsilon}$ is continuous at $t=0$.

For the sake of clear notation we introduce the following abbreviations:

$$
\begin{aligned}
{[u]_{\gamma, t, \alpha} } & :=\sup _{0 \leqslant s<s+h \leqslant t} \frac{\|u(s+h)-u(s)\|_{X^{\alpha / 2}}}{h^{\gamma}}, \\
{[\mathbf{V}]_{\gamma, t}: } & :=\sum_{i=1}^{N} \sup _{0 \leqslant s<s+h \leqslant t} \frac{\left|V_{i}(s+h)-V_{i}(s)\right|}{h^{\gamma}},
\end{aligned}
$$

where $t<T_{0}, \alpha \in\left[1, \frac{3}{2}\right), \gamma \in\left(0, \frac{1}{2}\right)$ and $\alpha+\gamma<\frac{3}{2}$. We also set

$$
\{u\}_{\gamma, t, \alpha}:=\sup _{0 \leqslant s \leqslant t} \frac{\left\|u(s)-u_{0}\right\|_{X^{\alpha / 2}}}{s^{\gamma}}, \quad\{\mathbf{V}\}_{\gamma, t}:=\sum_{i=1}^{N} \sup _{0 \leqslant s \leqslant t} \frac{\left|V_{i}(s)-V_{i}(0)\right|}{h^{\gamma}} .
$$

Of course, $\{u\}_{\gamma, t, \alpha} \leqslant[u]_{\gamma, t, \alpha},\{\mathbf{V}\}_{\gamma, t} \leqslant[\mathbf{V}]_{\gamma, t}$. The quantities $\{u\}_{\gamma, t, \alpha},\{\mathbf{V}\}_{\gamma, t}$ may be estimated using only initial data; the reason for their introduction is that they enter the estimates for $[u]_{\gamma, t, \alpha}$, $[\mathbf{V}]_{\gamma, t}$.

Let us first show how the representation (3.4) changes if we assume (3.1). Namely, after noting that $V_{i}^{\epsilon}(0)=V_{i}^{0}(0)$ we have

$$
\begin{aligned}
u^{\epsilon}(t)-u_{0} & =\mathrm{e}^{\Delta t / \epsilon} u_{0}-u_{0}-\frac{1}{\epsilon} \sum_{i=1}^{N} \int_{0}^{t} \Delta \mathrm{e}^{\Delta(t-\theta) / \epsilon} f_{i}\left(\mathbf{z}^{\epsilon}(\theta)\right) V_{i}^{\epsilon}(\theta) \mathrm{d} \theta \\
& =\frac{1}{\epsilon} \int_{0}^{t} \Delta \mathrm{e}^{\Delta(t-\theta) / \epsilon} \sum_{i=1}^{N}\left(f_{i}(0) V_{i}^{\epsilon}(0)-f_{i}\left(\mathbf{z}^{\epsilon}(\theta)\right) V_{i}^{\epsilon}(\theta)\right) \mathrm{d} \theta .
\end{aligned}
$$


We start with estimates on $\{u\}_{\gamma, t, \alpha}$ and $\{\mathbf{V}\}_{\gamma, t}$. Similar calculations will provide us with estimates of $[u]_{\gamma, t, \alpha}$ and $[\mathbf{V}]_{\gamma, t}$.

Lemma 4.1 Let us suppose that $s_{0}$ is convex, $Q\left(s_{0}\right)$ satisfies (3.7) and the numbers $\alpha \in\left[1, \frac{3}{2}\right)$, $\gamma \in\left(0, \frac{1}{2}\right), \delta \in\left(0, \frac{3}{4}-\frac{\alpha+\gamma}{2}\right)$ satisfy $\alpha+\gamma<\frac{3}{2}$, but otherwise they are arbitrary. Then, there exists a positive constant $K_{2}$, which is independent of $s_{0}$ and it has the following property. If we assume that $L^{\epsilon}(0)$ is small, meaning $s_{0}$ satisfies

$$
\sum_{i=1}^{N} \frac{K_{2}}{\beta_{i}}\left|\ln L^{\epsilon}(0) /(2 N \Lambda)\right|^{1 / 2} K_{0}(\lambda, \delta)\left(L^{\epsilon}(0) / 2\right)^{\gamma}<1
$$

as well as (3.9) and hypothesis (e) of [13: Theorem 10] are fulfilled with $K=\Lambda$, then for $t<T_{0}$

$$
\begin{aligned}
& \{u\}_{\gamma, t, \alpha} \leqslant C=C\left(s_{0}, \Omega, \lambda, \delta, \gamma, r\right) \\
& \{\mathbf{V}\}_{\gamma, t} \leqslant C=C\left(s_{0}, \Omega, \lambda, \delta, \gamma, r\right) .
\end{aligned}
$$

Proof. Let us note that due to (2.3a) and (2.3b) we have

$$
\begin{aligned}
V_{i}^{\epsilon}(t)-V_{i}^{\epsilon}(0)=\frac{1}{\beta_{i}} & \left(\frac{1}{L_{i}^{\epsilon}(t)}-\frac{1}{L_{i}^{\epsilon}(0)}\right)\left(\Gamma_{i}+\left(u_{0}, f_{i}(0)\right)_{H_{0}^{1}(\Omega)}\right) \\
& +\frac{1}{\beta_{i} L_{i}^{\epsilon}(t)}\left(u^{\epsilon}(t)-u_{0}, f_{i}\left(\mathbf{z}^{\epsilon}(t)\right)\right)_{H_{0}^{1}(\Omega)} \\
& +\frac{1}{\beta_{i} L_{i}^{\epsilon}(t)}\left(u_{0}, f_{i}\left(\mathbf{z}^{\epsilon}(t)\right)-f_{i}(0)\right)_{H_{0}^{1}(\Omega)} .
\end{aligned}
$$

Hence, by Corollary 3.9 and the definitions of $T_{0}$ and $B(0, r)$ in the previous section we have

$$
\begin{aligned}
& \frac{\left|V_{i}^{\epsilon}(t)-V_{i}^{\epsilon}(0)\right|}{t^{\gamma}} \leqslant \frac{C}{\beta_{i} t^{\gamma}}\left|\mathbf{z}^{\epsilon}(t)\right|\left(\left|\Gamma_{i}\right|+\left|\left(u_{0}, f_{i}(0)\right)_{H_{0}^{1}(\Omega)}\right|\right) \\
& \quad+\frac{1}{\beta_{i} L_{i}^{\epsilon}(t)}\left(\{u\}_{\gamma, t, \alpha}\left\|f_{i}\left(\mathbf{z}^{\epsilon}(t)\right)\right\|_{H_{0}^{1}(\Omega)}+C\left\|u_{0}\right\|_{H_{0}^{1}(\Omega)}\left|\mathbf{z}^{\epsilon}(t)\right|^{\gamma} t^{-\gamma}\right) .
\end{aligned}
$$

If we in addition use (3.5), (3.10) and Corollary 3.6, then we see

$$
\begin{aligned}
\frac{\left|V_{i}^{\epsilon}(t)-V_{i}^{\epsilon}(0)\right|}{t^{\gamma}} \leqslant & \frac{C N \Lambda|\mathbf{G}| t}{\beta_{i} t^{\gamma} L^{\epsilon}(t)}+\frac{C_{0}\{u\}_{\gamma, t, \alpha}}{\beta_{i}}\left|\ln L_{i}^{\epsilon}(t)\right|^{1 / 2} \\
& +\frac{C(N \Lambda)^{\gamma}\left\|u_{0}\right\|}{\beta_{i} L_{i}^{\epsilon}(t)\left(L^{\epsilon}(t)\right)^{\gamma}}|\mathbf{G}| .
\end{aligned}
$$

Finally, we can see that

$$
\begin{aligned}
\{\mathbf{V}\}_{\gamma, t} \leqslant & \frac{C N \Lambda t^{1-\gamma}}{L^{\epsilon}(t)}|\mathbf{G}| \sum_{i=1}^{N} \frac{1}{\beta_{i}}+C_{0}\{u\}_{\gamma, t, \alpha} \sum_{i=1}^{N} \frac{\left|\ln L_{i}^{\epsilon}(t)\right|^{1 / 2}}{\beta_{i}} \\
& +C(N \Lambda)^{1+\gamma} \sum_{i=1}^{N} \frac{\left\|u_{0}\right\|}{\beta_{i}} \frac{|\mathbf{G}|}{\left(L^{\epsilon}(t)\right)^{1+\gamma}} .
\end{aligned}
$$


Thus, the bound depends upon $\{u\}_{\gamma, t, \alpha}$, which we shall now determine. For this purpose we use (4.1). The combination of [8: 1.4.3], Corollary 3.9 and (3.6) yields

$$
\begin{aligned}
& \frac{\left\|u^{\epsilon}(t)-u_{0}\right\|_{X^{\alpha / 2}}}{t^{\gamma}} \\
& \leqslant \frac{1}{t^{\gamma} \epsilon} \int_{0}^{t}\left\|(-\Delta)^{1-\delta} \mathrm{e}^{\Delta(t-\theta) / \epsilon} \sum_{i=1}^{N}(-\Delta)^{\delta} f_{i}(0)\left(V_{i}^{\epsilon}(0)-V_{i}^{\epsilon}(\theta)\right)\right\|_{X^{\alpha / 2}} \mathrm{~d} \theta \\
& +\frac{1}{t^{\gamma} \epsilon} \int_{0}^{t}\left\|(-\Delta)^{1-\delta} \mathrm{e}^{\Delta(t-\theta) / \epsilon} \sum_{i=1}^{N}(-\Delta)^{\delta}\left(f_{i}(0)-f_{i}\left(\mathbf{z}^{\epsilon}(\theta)\right)\right) V_{i}^{\epsilon}(\theta)\right\|_{X^{\alpha / 2}} \mathrm{~d} \theta \\
& \leqslant \frac{C}{t^{\gamma} \epsilon} \int_{0}^{t}\left(\frac{t-\theta}{\epsilon}\right)^{-1+\delta} \mathrm{e}^{-\lambda(t-\theta) / \epsilon} \sum_{i=1}^{N}\left|V_{i}^{\epsilon}(\theta)-V_{i}^{\epsilon}(0)\right|\left\|f_{i}(0)\right\|_{X^{\alpha / 2}+\delta} \mathrm{d} \theta \\
& +\frac{C}{t^{\gamma} \epsilon} \int_{0}^{t}\left(\frac{t-\theta}{\epsilon}\right)^{-1+\delta} \mathrm{e}^{-\lambda(t-\theta) / \epsilon} \sum_{i=1}^{N}\left|\Gamma_{i}\right|\left|\mathbf{z}^{\epsilon}(\theta)\right|^{\gamma} /\left(\beta_{i} L_{i}^{\epsilon}(\theta)\right) \mathrm{d} \theta
\end{aligned}
$$

where $\delta>0$ is such that $\alpha+\gamma+2 \delta<\frac{3}{2}$ and $C$ depends only on $\Omega$ and $\delta$. We can now complete the calculation using (3.5) and the definition of $K_{0}(\lambda, \delta)$ :

$$
\{u\}_{\gamma, t, \alpha} \leqslant C_{2} K_{0}(\lambda, \delta)\left(\{\mathbf{V}\}_{\gamma, t}\left(L^{\epsilon}(0) / 2\right)^{\gamma}+|\mathbf{G}|_{1}|\mathbf{G}| \frac{(N \Lambda)^{1+\gamma}}{\left(L^{\epsilon}(t)\right)^{1+\gamma}}\right) .
$$

Interestingly, this estimate of $\{u\}_{\gamma, t, \alpha}$ in turn depends on $\{\mathbf{V}\}_{\gamma, t}$. We can solve the system of inequalities (4.3) and (4.4). For this purpose we insert (4.4) into (4.3). Simple calculations utilizing (3.10), Corollary 3.9, $K_{1}=C_{0} C_{2}$ and (4.2) lead us to the desired estimate on $\{\mathbf{V}\}_{\gamma, t}$ as well as on $\{u\}_{\gamma, t, \alpha}$.

We are now ready for the main estimates of this section. They are obtained by a method similar to that used in the previous proof. Here again formula (4.1) plays an important role.

Lemma 4.2 Let us suppose that the assumptions of the previous lemma hold, thus in particular $L^{\epsilon}(0)$ is small. Then, for $t<\inf _{\epsilon>0} T_{d}^{\epsilon}$

$$
[u]_{\gamma, t, \alpha} \leqslant C=C\left(s_{0}, \lambda, \Omega, \delta, \gamma, r\right), \quad[\mathbf{V}]_{\gamma, t} \leqslant C=C\left(s_{0}, \lambda, \Omega, \delta, \gamma, r\right) .
$$

Proof. We noted in Corollary 3.6 that $\left(L^{\epsilon}(t)\right)^{\prime}<0$. Hence we can define a sequence $\left\{T_{d, i}^{\epsilon}\right\}_{i=1}^{\infty}$ by formula

$$
T_{d, 0}^{\epsilon}=0, \quad T_{d, 1}^{\epsilon}=T_{0}
$$

and $T_{d, i+1}^{\epsilon}(i>1)$ is such that

$$
L^{\epsilon}\left(T_{d, i+1}^{\epsilon}\right)=\frac{1}{2} L^{\epsilon}\left(T_{d, i}^{\epsilon}\right) .
$$

Of course, for any $t<T_{0} \leqslant T_{\max }^{\epsilon}$, there is only a finite number of $T_{d, i}^{\epsilon} \mathrm{s}$ such that $T_{d, i}^{\epsilon}<t$, say $i=1, \ldots, p$. For, if it were otherwise, then at the accumulation point $T^{\star}$ of $T_{d, i}^{\epsilon} \mathrm{s}$ we would have $L^{\epsilon}\left(t^{\star}\right)=0$, which is impossible. 
We will split the estimates of $[u]_{\gamma, t, \alpha}$ and $[\mathbf{V}]_{\gamma, t}$ into a number of steps. In order to facilitate our calculations we write

$$
\begin{aligned}
& {[u]_{\gamma, t_{1}, t_{2}, \alpha}=\sup _{t_{1} \leqslant \tau<\tau+h \leqslant t_{2}} \frac{\|u(\tau+h)-u(\tau)\|_{X^{\alpha / 2}}}{h^{\gamma}}} \\
& {[\mathbf{V}]_{\gamma, t_{1}, t_{2}}=\sup _{t_{1} \leqslant \tau<\tau+h \leqslant t_{2}} \sum_{i=1}^{N} \frac{\left|V_{i}(\tau+h)-V_{i}(\tau)\right|}{h^{\gamma}} .}
\end{aligned}
$$

It is clear that

$$
\begin{aligned}
& {[u]_{\gamma, t, \alpha} \equiv[u]_{\gamma, 0, t, \alpha} \leqslant[u]_{\gamma, 0, T_{d, p}^{\epsilon}, \alpha}+[u]_{\gamma, T_{d, p}^{\epsilon}, t, \alpha}} \\
& {[\mathbf{V}]_{\gamma, t} \equiv[\mathbf{V}]_{\gamma, 0, t} \leqslant[\mathbf{V}]_{\gamma, 0, T_{d, p}^{\epsilon}}+[\mathbf{V}]_{\gamma, T_{d, p}^{\epsilon}, t} .}
\end{aligned}
$$

We will estimate $[u]_{\gamma, T_{d, i}^{\epsilon}, t, \alpha},[\mathbf{V}]_{\gamma, T_{d, i}^{\epsilon}, t}$ one after the other. We start with $[u]_{\gamma, t, \alpha}$ for $t \leqslant T_{d, 1}^{\epsilon}$, next we consider $[\mathbf{V}]_{\gamma, t}$. After a change of variables in the integral in (4.1) we arrive at

$$
\begin{aligned}
& u^{\epsilon}(t+h)-u^{\epsilon}(t) \\
& =\int_{0}^{(t+h) / \epsilon} \Delta \mathrm{e}^{\tau \Delta} \sum_{i=1}^{N}\left(f_{i}(0) V_{i}^{\epsilon}(0)-f_{i}\left(\mathbf{z}^{\epsilon}(t+h-\epsilon \tau)\right) V_{i}^{\epsilon}(t+h-\epsilon \tau)\right) \mathrm{d} \tau \\
& -\int_{0}^{t / \epsilon} \Delta \mathrm{e}^{\tau \Delta} \sum_{i=1}^{N}\left(f_{i}(0) V_{i}^{\epsilon}(0)-f_{i}\left(\mathbf{z}^{\epsilon}(t-\epsilon \tau)\right) V_{i}^{\epsilon}(t-\epsilon \tau)\right) \mathrm{d} \tau \\
& =\int_{0}^{t / \epsilon} \Delta \mathrm{e}^{\tau \Delta} \sum_{i=1}^{N} f_{i}\left(\mathbf{z}^{\epsilon}(t-\epsilon \tau)\right) V_{i}^{\epsilon}(t-\epsilon \tau) \mathrm{d} \tau \\
& \left.-\int_{0}^{t / \epsilon} \Delta \mathrm{e}^{\tau \Delta} \sum_{i=1}^{N} f_{i}\left(\mathbf{z}^{\epsilon}(t+h-\epsilon \tau)\right) V_{i}^{\epsilon}(t+h-\epsilon \tau)\right) \mathrm{d} \tau \\
& +\int_{t / \epsilon}^{(t+h) / \epsilon} \Delta \mathrm{e}^{\tau \Delta} \sum_{i=1}^{N}\left(f_{i}(0) V_{i}^{\epsilon}(0)-f_{i}\left(\mathbf{z}^{\epsilon}(t+h-\epsilon \tau)\right) V_{i}^{\epsilon}(t+h-\epsilon \tau)\right) \mathrm{d} \tau .
\end{aligned}
$$

We now estimate this difference divided by $h^{\gamma}$. We proceed in a similar fashion as we did during our estimates of $\{u\}_{\gamma, t, \alpha}$ :

$$
\begin{aligned}
& \frac{1}{h^{\gamma}}\left\|u^{\epsilon}(t+h)-u^{\epsilon}(t)\right\|_{X^{\alpha / 2}} \\
& =C \int_{0}^{t / \epsilon} \tau^{-1+\delta} \mathrm{e}^{-\lambda \tau}\left([\mathbf{V}]_{\gamma, t}\left(L^{\epsilon}(t-\epsilon \tau) / 2\right)^{\gamma}+\frac{|\mathbf{G}|_{1}|\mathbf{G}|^{\gamma}(N \Lambda)^{1+\gamma}}{\left(L^{\epsilon}(t+h)\right)^{1+\gamma}}\right) \mathrm{d} \tau \\
& \quad+\frac{C}{h^{\gamma}} \int_{t / \epsilon}^{(t+h) / \epsilon} \mathrm{e}^{-\lambda \tau} \frac{(t+h-\epsilon \tau)^{\gamma}}{\tau^{1-\delta}}\{\mathbf{V}\}_{\gamma, t}\left(L^{\epsilon}(0) / 2\right)^{\gamma} \mathrm{d} \tau \\
& \quad+\frac{C}{h^{\gamma}} \int_{t / \epsilon}^{(t+h) / \epsilon} \mathrm{e}^{-\lambda \tau} \frac{(t+h-\epsilon \tau)^{\gamma}}{\tau^{1-\delta}} \frac{|\mathbf{G}|_{1}|\mathbf{G}|(N \Lambda)^{1+\gamma}}{\left(L^{\epsilon}(t+h)\right)^{1+\gamma}} \mathrm{d} \tau
\end{aligned}
$$


where $\delta \in\left(0, \frac{3}{4}-(\alpha+\gamma) / 2\right)$. We apply the Second Mean Theorem to the second integral and the third one. We then arrive at

$$
\begin{aligned}
& \frac{1}{h^{\gamma}}\left\|u^{\epsilon}(t+h)-u^{\epsilon}(t)\right\|_{X^{\alpha / 2}} \\
& \leqslant C K_{0}(\lambda, \delta)\left([\mathbf{V}]_{\gamma, t}\left(L^{\epsilon}(0) / 2\right)^{\gamma}+|\mathbf{G}|_{1}|\mathbf{G}| \frac{(N \Lambda)^{1+\gamma}}{\left(L^{\epsilon}(t+h)\right)^{1+\gamma}}\right) \\
& +C \int_{t / \epsilon}^{\xi} \tau^{-1+\delta} \mathrm{e}^{-\lambda \tau} \mathrm{d} \tau\left(\{\mathbf{V}\}_{\gamma, t}\left(L^{\epsilon}(0) / 2\right)^{\gamma}+|\mathbf{G}|_{1}|\mathbf{G}| \frac{(N \Lambda)^{1+\gamma}}{\left(L^{\epsilon}(t+h)\right)^{1+\gamma}}\right),
\end{aligned}
$$

where $\xi$ is a number in $[t / \epsilon,(t+h) / \epsilon]$. Finally,

$$
\begin{aligned}
{[u]_{\gamma, t, \alpha} \leqslant } & C_{2} K_{0}(\lambda, \delta)\left(L^{\epsilon}(0) / 2\right)^{\gamma}\left([\mathbf{V}]_{\gamma, t}+\{\mathbf{V}\}_{\gamma, t}\right) \\
& +C K_{0}(\lambda, \delta)|\mathbf{G}|_{1}|\mathbf{G}| \frac{(N \Lambda)^{1+\gamma}}{\left(L^{\epsilon}(t+h)\right)^{1+\gamma}} .
\end{aligned}
$$

As before the estimate on $[u]_{\gamma, t, \alpha}$ depends upon $[\mathbf{V}]_{\gamma, t}$. We shall now find the latter. If we mimic the calculations for $\{\mathbf{V}\}_{\gamma, t}$, we find that

$$
\begin{aligned}
& \frac{\left|V_{i}^{\epsilon}(t+h)-V_{i}^{\epsilon}(t)\right|}{h^{\gamma}} \\
& \leqslant \frac{C}{\beta_{i} h^{\gamma}}\left|\mathbf{z}^{\epsilon}(t+h)-\mathbf{z}^{\epsilon}(t)\right|\left(\left|\Gamma_{i}\right|+C\left\|u^{\epsilon}(t)\right\|_{H_{0}^{1}(\Omega)} L_{i}^{\epsilon}(t)\left|\ln L_{i}^{\epsilon}(t)\right|^{1 / 2}\right) \\
& \quad+\frac{C_{0} L_{i}^{\epsilon}(t)}{\beta_{i} L_{i}^{\epsilon}(t) h^{\gamma}}\left|\ln L_{i}^{\epsilon}(t)\right|^{1 / 2}\left\|u^{\epsilon}(t+h)-u^{\epsilon}(t)\right\|_{H_{0}^{1}(\Omega)} \\
& \quad+\left\|u^{\epsilon}(t)\right\|_{H_{0}^{1}(\Omega)} \frac{\left\|f_{i}\left(\mathbf{z}^{\epsilon}(t+h)\right)-f_{i}\left(\mathbf{z}^{\epsilon}(t)\right)\right\|_{H_{0}^{1}(\Omega)}}{\beta_{i} L_{i}^{\epsilon}(t+h) h^{\gamma}} .
\end{aligned}
$$

At this point we need uniform estimates for $\max _{t \in\left[0, T_{0}\right]}\left\|u^{\epsilon}(t)\right\|_{H_{0}^{1}(\Omega)}$, but they follow from the uniform estimates for $[u]_{\gamma, t, \alpha}$ and the fact that $u^{\epsilon}(0)=u_{0}$ for all $\epsilon>0$. Hence,

$$
\max _{t \in\left[0, T_{d, 1}^{\epsilon}\right]}\left\|u^{\epsilon}(t)\right\|_{H_{0}^{1}(\Omega)}=K\left(T_{d, 1}^{\epsilon}\right)<\infty .
$$

Continuing our estimates we obtain

$$
\begin{aligned}
& \frac{\left|V_{i}^{\epsilon}(t+h)-V_{i}^{\epsilon}(t)\right|}{h^{\gamma}} \\
& \leqslant \frac{C N \Lambda t}{\beta_{i} L^{\epsilon}(t)}|\mathbf{G}| h^{1-\gamma}\left(\left|\Gamma_{i}\right|+K\left(T_{d, 1}^{\epsilon}\right) L_{i}^{\epsilon}(t)\left|\ln L_{i}^{\epsilon}(t)\right|^{1 / 2}\right) \\
& \quad+\frac{C_{0}}{\beta_{i}}[u]_{\gamma, t, \alpha}\left|\ln L_{i}^{\epsilon}(t+h)\right|^{1 / 2}+\frac{C|\mathbf{G}|(N \Lambda)^{\gamma} K\left(T_{d, 1}^{\epsilon}\right)}{\beta_{i} L_{i}^{\epsilon}(t+h)\left(L^{\epsilon}(t+h)\right)^{\gamma}} .
\end{aligned}
$$

At this point we see that again we obtain a system of two inequalities for $[u]_{\gamma, t, \alpha}$ and $[\mathbf{V}]_{\gamma, t}$. After 
we insert (4.5) into the above inequality we find that

$$
\begin{aligned}
{[\mathbf{V}]_{\gamma, t} } & \leqslant \sum_{i=1}^{N} \frac{C N \Lambda|\mathbf{G}|\left(T_{d, 1}^{\epsilon}\right)^{1-\gamma}}{\beta_{i} L^{\epsilon}\left(T_{d, 1}^{\epsilon}\right)}\left(\left|\Gamma_{i}\right|+K\left(T_{d, 1}^{\epsilon}\right) \frac{L^{\epsilon}\left(T_{d, 1}^{\epsilon}\right)}{N \Lambda}\left|\ln \frac{L^{\epsilon}\left(T_{d, 1}^{\epsilon}\right)}{N \Lambda}\right|^{1 / 2}\right) \\
& +\sum_{i=1}^{N} \frac{K_{2}}{\beta_{i}}\left|\ln \frac{L^{\epsilon}\left(T_{d, 1}^{\epsilon}\right)}{2 N \Lambda}\right|^{1 / 2} K_{0}(\lambda, \delta)\left(L^{\epsilon}(0) / 2\right)^{\gamma}\left([\mathbf{V}]_{\gamma, t}+\{\mathbf{V}\}_{\gamma, t}\right) \\
& +\sum_{i=1}^{N} \frac{C}{\beta_{i}}\left|\ln L^{\epsilon}\left(T_{d, 1}^{\epsilon}\right) /(N \Lambda)\right|^{1 / 2} K_{0}(\lambda, \delta)|\mathbf{G}|_{1}|\mathbf{G}| \frac{(N \Lambda)^{1+\gamma}}{\left(L^{\epsilon}\left(T_{d, 1}^{\epsilon}\right)\right)^{1+\gamma}} \\
& +\sum_{i=1}^{N} \frac{C(N \Lambda)^{1+\gamma} K\left(T_{d, 1}^{\epsilon}\right)}{\beta_{i}\left(L^{\epsilon}\left(T_{d, 1}^{\epsilon}\right)\right)^{1+\gamma}}|\mathbf{G}| .
\end{aligned}
$$

We can proceed if we recall the definition of $T_{d, 1}^{\epsilon}: L^{\epsilon}\left(T_{d, 1}^{\epsilon}\right)=\frac{1}{2} L^{\epsilon}(0)$. Now, the assumption (4.2) implies that this inequality is solvable for $[\mathbf{V}]_{\gamma, t}$. We conclude that

$$
[\mathbf{V}]_{\gamma, t} \leqslant K=K\left(L^{\epsilon}(0), N, \mathbf{G}, \mathbf{B}, \Omega, \lambda, \delta\right)<\infty, \quad t \leqslant T_{d, 1}^{\epsilon}
$$

independently of $\epsilon>0$. This estimates combined with (4.5) yield the desired estimate for $[u]_{\gamma, t, \alpha}$, provided that $t \leqslant T_{d, 1}^{\epsilon}$.

Subsequently, we proceed by induction. Assuming we have established bounds for $[u]_{\gamma, 0, T_{d, i}^{\epsilon}, \alpha}$, $[\mathbf{V}]_{\gamma, 0, T_{d, i}^{\epsilon}}$ we work on $[u]_{\gamma, T_{d, i}^{\epsilon}, t, \alpha}$, and $[\mathbf{V}]_{\gamma, T_{d, i}^{\epsilon}, t}$, where $t \leqslant T_{d, i+1}^{\epsilon}$.

We note that calculations leading to (4.5) yield also

$$
\begin{aligned}
{[u]_{\gamma, T_{d, i}^{\epsilon}, t, \alpha} \leqslant } & C_{0} K_{0}(\lambda, \delta)\left(L^{\epsilon}(0) / 2\right)^{\gamma}\left([\mathbf{V}]_{\gamma, T_{d, i}^{\epsilon}, t}+[\mathbf{V}]_{\gamma, 0, T_{d, i}^{\epsilon}}+\{\mathbf{V}\}_{\gamma, t}\right) \\
& +C K_{0}(\lambda, \delta)|\mathbf{G}|_{1}|\mathbf{G}| \frac{(N \Lambda)^{1+\gamma}}{\left(L^{\epsilon}(t+h)\right)^{1+\gamma}} .
\end{aligned}
$$

On the other hand, the calculations leading to (4.6) provide us with

$$
\begin{aligned}
& {[\mathbf{V}]_{\gamma, T_{d, i}^{\epsilon}, t}} \\
& \quad \leqslant \sum_{j=1}^{N} \frac{C N \Lambda|\mathbf{G}| h^{1-\gamma}}{\beta_{j} L^{\epsilon}\left(T_{d, i+1}^{\epsilon}\right)}\left(\left|\Gamma_{i}\right|+K\left(T_{d, i+1}^{\epsilon}\right) \frac{L^{\epsilon}\left(T_{d, i+1}^{\epsilon}\right)}{N \Lambda}\left|\ln L^{\epsilon} \frac{\left(T_{d, i+1}^{\epsilon}\right)}{N \Lambda}\right|^{1 / 2}\right) \\
& \quad+\sum_{j=1}^{N} \frac{C_{2}}{\beta_{j}}[u]_{\gamma, T_{d, i}^{\epsilon}, t, \alpha}\left|\ln \left(L^{\epsilon}\left(T_{d, i+1}^{\epsilon}\right) / 2 N \Lambda\right)\right|^{1 / 2} \\
& \quad+\sum_{j=1}^{N} \frac{C(N \Lambda)^{1+\gamma} K\left(T_{d, i+1}^{\epsilon}\right)}{\beta_{j}\left(L^{\epsilon}(t+h)\right)^{1+\gamma}}|\mathbf{G}| .
\end{aligned}
$$

Using the same reasoning as we did for estimating $[u]_{\gamma, t, \alpha},[\mathbf{V}]_{\gamma, t}$ we conclude the proof.

The bounds we have established above are sufficient to prove convergence of solutions and we will now describe our main theorem. 
THEOREM 4.3 Let us assume that $\alpha \in\left[1, \frac{3}{2}\right), \gamma \in\left(0, \frac{1}{2}\right), \alpha+\gamma<\frac{3}{2}$ and $u^{\epsilon}(0)=u_{0}$, where $u_{0}$ is given by (3.1). We assume that the initial interface $s_{0}$ is convex, $Q\left(s_{0}\right)$ satisfies (3.7) and $s_{0}$ is small, i.e. hypothesis (e) of [13: Theorem 10] is fulfilled with $K=\Lambda$, and (3.9) and (4.2) hold. If $\left(\mathbf{z}^{\epsilon}, u^{\epsilon}\right)$ is a unique solution to $\left(\mathrm{P}_{\epsilon}\right)$, then for any $0<T<\inf _{\epsilon>0} T_{\max }^{\epsilon}$

(a) $V_{i}^{\epsilon}$ converges to $V_{i}^{0}$ in $C^{0, \gamma}([0, T]), i=1, \ldots, N$, hence $\mathbf{z}^{\epsilon}$ converges to $\mathbf{z}^{0}$ in $C^{1, \gamma}([0, T])$;

(b) $u^{\epsilon}$ converges to $u^{0}$ in $C^{0, \gamma}\left([0, T] ; X^{\alpha / 2}\right)$ and $\left(\mathbf{z}^{0}, u^{0}\right)$ is a unique solution to $\left(\mathrm{P}_{0}\right)$.

Proof. Let us consider any sequence $0<\epsilon_{k}$ converging to zero. By Lemma 4.2 the sequence $V_{i}^{\epsilon_{k}}$ is bounded in $C^{0, \gamma^{\prime}}([0, T])$ for any $\gamma^{\prime} \in\left(0, \frac{1}{2}\right)$. So, it is possible to extract a subsequence (still denoted by $\left.V_{i}^{\epsilon_{k}}\right)$ converging in $C^{0, \gamma}([0, T]), 0<\gamma<\gamma^{\prime}$ to some $V_{i}^{\infty}$. Hence, $\mathbf{z}^{\epsilon_{k}}$ converges to $\mathbf{z}^{\infty}$ in $C^{1, \gamma}([0, T])$. Similarly, we can show that for $\alpha<\frac{3}{2}$

$$
u^{\epsilon_{k}} \rightarrow u^{\infty} \quad \text { in } \quad C^{0, \gamma}\left([0, T] ; X^{\alpha / 2}\right) .
$$

We shall now show that $\left(\mathbf{z}^{\infty}, u^{\infty}\right)$ is a solution to $\left(\mathrm{P}_{0}\right)$. It is easy to see that

$$
V_{i}^{\infty}=\left(\Gamma_{i}-\left(u^{\infty}, f_{i}\left(\mathbf{z}^{\infty}\right)\right)_{H_{0}^{1}(\Omega)}\right) / \beta_{i} L_{i}^{\infty}(t) .
$$

and we will show that

$$
u^{\infty}(t)=\sum_{i=1}^{N} f_{i}\left(\mathbf{z}^{\infty}(t)\right) V_{i}^{\infty}(t) .
$$

For this purpose we will show that $I:=u^{\epsilon_{k}}(t)-\sum_{i=1}^{N} f_{i}\left(\mathbf{z}^{\epsilon_{k}}(t)\right) V_{i}^{\epsilon_{k}}(t)$ converges to zero in $C^{0, \gamma}\left([0, T] ; X^{\alpha / 2}\right)$. We have

$$
\begin{aligned}
I= & \mathrm{e}^{\Delta t / \epsilon_{k}}\left(\sum_{i=1}^{N} f_{i}\left(\mathbf{z}^{\epsilon_{k}}(0)\right) V_{i}^{\epsilon_{k}}(0)-f_{i}\left(\mathbf{z}^{\epsilon_{k}}(t)\right) V_{i}^{\epsilon_{k}}(t)\right) \\
& +\int_{0}^{t / \epsilon_{k}} \Delta \mathrm{e}^{\Delta \tau} \sum_{i=1}^{N}\left(f_{i}\left(\mathbf{z}^{\epsilon_{k}}\left(t-\epsilon_{k} \tau\right)\right) V_{i}^{\epsilon_{k}}\left(t-\epsilon_{k} \tau\right)-f_{i}\left(\mathbf{z}^{\epsilon_{k}}(t)\right) V_{i}^{\epsilon_{k}}(t)\right) .
\end{aligned}
$$

Hence, for any $\left.0<\delta<\left(\frac{3}{4}-(\alpha+\gamma) / 2\right)\right)$ we have

$$
\begin{aligned}
\|I\|_{X^{\alpha / 2}} \leqslant & C \mathrm{e}^{-\lambda t / \epsilon_{k}}\left\|u_{0}-\sum_{i=1}^{N} f_{i}\left(\mathbf{z}^{\epsilon_{k}}(t)\right) V_{i}^{\epsilon_{k}}(t)\right\|_{X^{\alpha / 2}} \\
& +\int_{0}^{t / \epsilon_{k}} \tau^{-1+\delta} \mathrm{e}^{-\lambda \tau}[\mathbf{V}]_{\gamma, t}^{\epsilon_{k}}\left(\epsilon_{k} \tau\right)^{\gamma} \max _{i=1, \ldots, N}\left(L_{i}^{\epsilon_{k}}(t)\right)^{\gamma} \mathrm{d} \tau \\
& +\int_{0}^{t / \epsilon_{k}} \tau^{-1+\delta} \mathrm{e}^{-\lambda \tau} C\left|V_{i}^{\epsilon_{k}}\left(t-\epsilon_{k} \tau\right) \| \mathbf{z}^{\epsilon_{k}}\left(t-\epsilon_{k} \tau\right)-\mathbf{z}^{\epsilon_{k}}(t)\right|^{\gamma} \mathrm{d} \tau .
\end{aligned}
$$

We note that $\left\|f_{i}\left(\mathbf{z}^{\epsilon_{k}}(t)\right)\right\|_{X^{\alpha / 2}}$ and $V_{i}^{\epsilon_{k}}(t), i=1, \ldots, N$ are uniformly bounded on $[0, T]$ due to Lemma 4.1. Because of the uniform convergence of $V_{i}^{\epsilon_{k}}$ it follows that

$$
\lim _{k \rightarrow \infty} V_{i}^{\epsilon_{k}}\left(t-\epsilon_{k} \tau\right)=V_{i}^{\infty}(t), \quad \lim _{k \rightarrow \infty} \mathbf{z}^{\epsilon_{k}}\left(t-\epsilon_{k} \tau\right)=\mathbf{z}^{\infty}(t) .
$$


In this way we may conclude that $\left\|u^{\epsilon_{k}}(t)-\sum_{i=1}^{N} f_{i}\left(\mathbf{z}^{\epsilon_{k}}(t)\right) V_{i}^{\epsilon_{k}}(t)\right\|_{X^{\alpha / 2}}$ goes to zero as $k \rightarrow \infty$. Equivalently, it means

$$
u^{\infty}(t)=\sum_{i=1}^{N} V_{i}^{\infty}(t) f_{i}\left(\mathbf{z}^{\infty}\right),
$$

or the pair $\left(\mathbf{z}^{\infty}, u^{\infty}\right)$ is a (unique) solution to $\left(\mathrm{P}_{0}\right)$. This shows that the only accumulation point of any sequence $\left(\mathbf{z}^{\epsilon_{k}}, V_{i}^{\epsilon_{k}}\right)$ is $\left(\mathbf{z}^{0}, V_{i}^{0}\right)$ so we can write

$$
\lim _{\epsilon \rightarrow 0} V_{i}^{\epsilon}=V_{i}^{0}, \quad \lim _{\epsilon \rightarrow 0} \mathbf{z}^{\epsilon}=\mathbf{z}^{0}, \quad \lim _{\epsilon \rightarrow 0} u^{\epsilon}=u^{0}
$$

\section{Acknowledgement}

The author wishes to thank Professor Y. Giga for stimulating discussions on the subject of this paper. The research for this paper was in part supported by the KBN grant 2 P03A 03517 . The author is also indebted to the referees for their remarks which led to an improvement of the text and the removal some inaccuracies.

\section{REFERENCES}

1. Almgren, F. \& WANG, L. Mathematical existence of crystal growth with Gibbs-Thomson curvature effects. J. Geom. Anal. 10, (2000) 1-100.

2. Ben Amar, \& PomeaU, Y. Growth of faceted needle crystals: theory. Europhys. Lett. 6, (1988) 609614.

3. Bellettini, G., Novaga, M., \& PaOlini, M. Facet-breaking for three-dimensional crystals evolving by mean curvature. Interfaces and Free Boundaries 1, (1999) 39-56.

4. Chen, X. \& Reitich, F. Local existence and uniqueness of solutions to the Stefan problem with surface tension and kinetic undercooling. J. Math. Anal. Appl. 164, (1992) 350-362.

5. GigA, M.-H. \& GigA, Y. A subdiffential interpretation of crystalline motion under nonuniform driving force. In: Chen, W. \& HU, S. (eds), Proc. International Conference on Dynamical Systems and Differential Equations, vol. I. pp. 276-287. Southwest Missouri State University, Springfield, MO (1998).

6. Gurtin, M. Thermomechanics of Evolving Phase Boundaries in the Plane. Clarendon Press, Oxford (1993).

7. Gurtin, M. \& Matias, J. Thermomechanics and the formulation of the Stefan problem for fully faceted interfaces. Quart. Appl. Math. 53, (1995) 761-782.

8. HENRY, D. Geometric theory of semilinear parabolic equations. Lecture Notes in Mathematics, 840. Springer, Berlin (1981) (Russian translation: Mir, Moscow 1985).

9. Herring, C. Surface tension as a motivation for sintering. In: Kingston, W. E. (ed), The Physics of Powder Metallurgy. McGraw-Hill, New York (1958).

10. LuCKHAUS, S. Solutions for the two-phase Stefan problem with the Gibbs-Thomson law for the melting temperature. Euro. J. Appl. Math. 1, (1990) 101-111.

11. RADKEVICH, E. V. The Gibbs-Thomson correction and condition for the existence of classical solution of the modified Stefan problem. Soviet Math. Dokl. 43, (1991) 274-278.

12. RYBKA, P. A quasi-steady approximation to an integro-differential model of interface motion. Appl. Anal. 56, (1995) 19-34.

13. RYBKA, P. A crystalline motion: uniqueness and geometric properties. SIAM J. Appl. Math. 57, (1997) 53-72. 
14. RYBKA, P. Crystalline version of the Stefan problem with Gibbs-Thomson law and kinetic undercooling. Adv. Differential Equations 3, (1998) 687-713.

15. RYBKA, P. The crystalline version of the modified Stefan problem in the plane and its properties. SIAM J. Math. Anal. 30, (1999) 756-786. (electronic).

16. Soner, H. M. Convergence of the phase-field equations to the Mullins-Sekerka problem with kinetic undercooling. Arch. Ration. Mech. Anal. 131, (1995) 134-197.

17. TAYLOR, J. E. Motion of curves by crystalline curvature, including triple junctions and boundary points. Proc. Symp. Pure Math. 54, (1993) 417-438. 